\title{
Simple partial status epilepticus: causes, treatment, and outcome in 47 patients
}

\author{
Frans B Scholtes, Willy O Renier, Harry Meinardi
}

\begin{abstract}
A retrospective case note review was conducted of 47 patients of 15 years and older who had sustained simple partial status epilepticus (SPSE) in The Netherlands between 1980 and 1987. In 46 patients the type of SPSE was somatomotor (in four adversive), and in one aphasic with visual and auditory hallucinations. SPSE was more common over the age of 50 . Six of 27 patients with previous epilepsy had an acute symptomatic cause. In 20 patients without previous epilepsy stroke was the most frequent cause (75\%). Outcome was determined by the underlying cause. In one patient the continuing epileptic activity may have caused neuronal damage.
\end{abstract}

(F Neurol Neurosurg Psychiatry 1996;61:90-92)

Keywords: simple partial status epiepticus; causes; treatment; outcome

Simple partial status epilepticus (SPSE) is characterised by partial seizures without impairment of consciousness or secondary generalisation, and with preserved neurovegetative regulation. ${ }^{1}$ The clinical expression depends on the region of the brain where the seizures originate.The most frequent type of SPSE is somatomotor. Other types include aphasic and somatosensory SPSE. Partial seizures with preserved consciousness are called SPSE when continuous clinical and EEG seizures are present for at least $30 \mathrm{~min}$ utes. $^{2}$

In general neurological practice two groups of patients with SPSE are encountered most often:

(1) Those with a succession of simple partial seizures with Jacksonian march, without persistent segmental myoclonus (somatomotor SPSE).

(2) Those with persistent myoclonus in a limited area of the body, present for weeks or months and sometimes in combination with somatomotor or tonic-clonic seizures (epilepsia partialis continua, EPC). Consciousness may be normal but can also be impaired.

Both types are usually caused by a symptomatic brain lesion, but may also occur in patients with a history of epilepsy.
Somatomotor SPSE is synonymous with EPC when myoclonic jerks are continuously present in the same body parts affected by the somatomotor seizures. ${ }^{2}{ }^{4}$ Most case studies or reviews concern EPC; other types of SPSE have only rarely been described..$^{4-8}$ As part of a study on status epilepticus in The Netherlands we were able to study retrospectively 47 adult patients with SPSE. We were particularly interested in the various types of SPSE, the causes, EEG findings, treatment, and outcome.

\section{Methods}

The cooperation of neurologists in 50 different hospitals in The Netherlands was requested. Fourteen agreed to cooperate, but two were unable to provide adequate documentation of admissions and discharges. In addition data from two of the three epilepsy centres in The Netherlands were included. We restricted this retrospective study to adult patients (older than 15 years).

During the period $1980-87$ we gathered a total of 576 admissions caused by various types of SE. We visited every hospital and inspected the patient files.

For SPSE, only patients with preserved consciousness and with a minimum seizure duration of 30 minutes were included. Patients with hemi-grand mal SE, asymmetric generalised tonic-clonic $\mathrm{SE}$, or patients with successive secondary generalised tonic-clonic seizures with persistent clonic jerks of one side of the body and impaired level of consciousness were excluded.

When a single tonic-clonic seizure preceded or occurred at the end of the SPSE, the patient was included. Morbidity was defined as all new neurological signs occurring during the period of SE and was calculated per event. The causes of morbidity were classified as $(a)$ the underlying cause, $(b)$ the seizures themselves and the ensuing medical complications, and $(c)$ unknown (when the contribution of the underlying cause or the seizures themselves could not exactly be established). The causes of mortality have been divided in the same way.

Outcome has been described as the neurological condition at discharge from the hospital. 
Treatment was regarded as inadequate when an insufficient dose was given, when the route of treatment was wrong (for example, intramuscular injection of diazepam), when unnecessary delay was present (for example, waiting for more than an hour after diazepam injection while the seizure continued before giving a second agent), when mechanical ventilation was not started despite signs of respiratory failure or the presence of various other medical complications, or when EEG monitoring was not done on patients treated with antiepileptic drugs together with curarisation.

\section{Results}

The Netherlands has a population of nearly 15 million people. About 100000 have epilepsy. The admissions we retrieved covering a period of eight years concerned 47 patients; 27 were known to have a history of epilepsy. The causes of previous epilepsy were symptomatic in 18 , whereas in nine patients the cause remained unknown. In five patients SPSE ended with a generalised tonic-clonic seizure, in seven a tonic-clonic seizure preceded SPSE.

In our material a preponderance of 30 women versus 17 men was found. There was an increase of SPSE with age. Only 16 of our patients were younger than 50 ; two were younger than 30 .

TYPES OF SPSE

Apart from one patient with aphasic SE all showed somatomotor SPSE; four of these had adversive SPSE.

The facial musculature was involved in 38 patients, alone (eight) or in combination with the eyes (one), an arm (seven), platysma (two), and an arm and a leg (20). In four patients jerking was limited to an arm (one), a hand (two), or the abdominal wall (one).

The patient with aphasic SPSE was a woman aged 67, known to have had previous epilepsy (caused by stroke), who developed SPSE with a duration of five days. The clinical presentation consisted of aphasia, acoustic hallucinations (strange sounds, music, voices), left sided headache, and visual hallucinations (red balls in the front of the right eye). She remained alert and showed recurrent epileptic seizures on the EEG, starting in the left occipital region with a $13 \mathrm{~Hz}$ sharp wave activity, changing to spike wave activity (3-4 Hz), and spreading over the left hemisphere. Sometimes a start was seen in the left paracentral region. Further investigations could not establish a cause. When the EEG showed that the SPSE had been arrested the patient continued to exhibit persistent word finding problems and a slight verbal memory deficit.

In 26 of 46 patients with somatomotor SPSE, frank seizures were evident without documented continuous myoclonus. The remaining 20 , showed more or less continuous clonic or myoclonic jerks, corresponding to EPC. When we compared the patients with EPC with those without continuous myoclonus, no differences were found for outcome, number of patients with previous epilepsy, or causes.
During SPSE EEG investigation was made in only 13 patients and showed abnormal findings corresponding to the clinical expression in 12 of them. However, specific epileptic discharges were present in only five patients. Brain CT was performed in all; MRI, PET, or SPECT in none.

\section{CAUSES OF SPSE}

In 27 patients with previous epilepsy six developed SPSE because of a new neurological problem: metastases (two), stroke (one), pneumococcal meningitis (one), osteomyelitis of the skull (one), and hypocalcaemia (one). Compliance problems (antiepileptic drugs) were only present in three patients. In three patients a systemic infection was considered to be the precipitating factor. In 15 patients the cause remained unknown.

In 20 patients without previous epilepsy stroke seemed the most prominent cause (14). In three patients a brain tumour had been found, whereas in another three patients a cause could not be established.

DURATION OF SPSE

Duration was less than two hours in 13 patients, between two and four hours in 12 , between four and 20 hours in four, and longer than 20 hours in 18. Half of these had a duration of one to three days, the other half between three and 14 days. An exceptionally long duration (seven weeks or more than one year) was seen in three patients with continuous focal myoclonic jerks (EPC).

\section{OUTCOME OF SPSE}

Four patients died, all due to cerebral infarction.

Morbidity after SPSE was present in 10 patients and consisted of paresis (four), aphasia (two), psychiatric disturbance (one), cognitive impairment (two), and persistent impairment of consciousness (one). Morbidity was mainly due to the underlying cause (six patients); in three patients the exact cause of morbidity could not be established. In one patient morbidity was attributed to SE itself.

In the six patients with sequelae because of the underlying cause, four had had a cerebral infarction, one a subdural haematoma, and one an intracerebral haematoma. The patients with sequelae of unknown cause had all had previous epilepsy.

\section{TREATMENT}

The last treatment, given just before SPSE ended or given as a last attempt consisted mainly of benzodiazepines, in particular $(40 \%)$ clonazepam or clonazepam with phenytoin. Other drugs were intravenous phenytoin, diazepam, or lidocaine, etomidate infusion, thiopentone infusion, carbamazepine, and intravenous calcium in a case of hypocalcaemia. In this last case calcium was immediately successful, whereas diazepam and clonazepam failed.

When outcome was related to quality of treatment we found in patients with good outcome that treatment was inadequate in $21 \%$; 
in patients with morbidity in $40 \%$, and in patients with fatal outcome in $50 \%$.

\section{Discussion}

The symptom SPSE is relatively rare and most case reports and reviews concern EPC, also named somatomotor SPSE. ${ }^{4}$ We did not find specific differences between patients with continuous clonic or myoclonic activity and patients with partial motor seizures for cause, outcome, and the presence of previous epilepsy. Our findings differ from those published for the frequency of previous epilepsy in patients with SPSE. Duration of SPSE in our series was generally shorter than mentioned in the medical literature and we differ in having a higher prevalence of female patients.

The cause of SPSE in our 20 patients without previous epilepsy was stroke in $70 \%$, a brain tumour in $15 \%$, and unknown in another $15 \%$. Two of the latter patients had a chronic psychiatric disease and one a remote symptomatic cause (subarachnoid haemorrhage some years earlier).

In patients with previous epilepsy an acute neurological problem seemed to be a prominent cause $(22 \%)$.

No correlation could be established between outcome and duration. Outcome was mainly determined by the underlying cause. In three patients the cause of morbidity remained unknown. All three had previous epilepsy; one was suspected to have a brain lesion, but the exact nature was not known; another was mentally retarded and the third had epilepsy because of stroke and a new cerebrovascular accident was suggested but this could not be determined with certainty. Only in one case SPSE itself as a cause of morbidity was likely.

Continuing seizure activity as a cause of morbidity in PSE has been established in complex partial SE, ${ }^{9}$ and SPSE as a cause of morbidity has been suggested in some other patients. ${ }^{10-11}$

In SPSE, as in $\mathrm{GCSE}^{12}$ we found that treatment was more often inadequate in patients with less good outcome, but the number of patients with mortality or morbidity was too low to draw conclusions.

Treatment of SE, irrespective of type, should be according to protocol. ${ }^{13} \mathrm{We}$ advise starting with rectal diazepam solution, 20-30 $\mathrm{mg}$ in adult patients. When seizures persist for 10 minutes after the diazepam give $1 \mathrm{mg}$ clon- azepam intravenously. Next the patient should be transferred to the intensive care unit because of the threat of respiratory depression. Continue with $1 \mathrm{mg}$ clonazepam every one or two minutes intravenously until seizures stop or respiratory depression becomes evident. If clonazepam fails after 10 minutes, start phenytoin-sodium intravenously $(15-18 \mathrm{mg} / \mathrm{kg}$ with a rate of $50 \mathrm{mg} / \mathrm{min}$ ). For those who continue with seizures start with midazolam-infusion at a rate of $0.3-0.6 \mathrm{mg} / \mathrm{kg} / 24 \mathrm{~h}$ after a bolus injection of $0.1-0.3 \mathrm{mg} / \mathrm{kg}$. We also have good results with propofol infusion. In most patients with SPSE thiopentone infusion is not necessary.

During treatment adequate medical care with special attention to respiration is essential. The cause of SPSE should be established as soon as possible, maintenance treatment with antiepileptic drugs should be continued or started. In patients resistant to drug therapy surgical treatment may be considered. ${ }^{14}$

We thank Mrs G van Etten for preparing the manuscript.

1 Gastaut H. Classification of status epilepticus. In: DelgadoEscueta A, Treiman D, Wasterlain C, Porter R, eds. Status epilepticus. Advances in neurology. Vol 34. New York: Raven Press, 1983:15-47.

2 Delgado-Escueta A, Treiman D. Focal status epilepticus: modern concepts. In: Lüders H, Lesser R, eds. Epilepsy: electroclinical syndromes. New York: Springer-Verlag, 1987:347-93.

3 Thomas J, Reagan T, Klass D. Epilepsia partialis continua Arch Neurol 1977; 34:266-75.

4 Schomer D. Focal status epilepticus and epilepsia partialis continua in adults and children. Epilepsia 1993;34(suppl 1):S29-36.

5 Löhler J, Peters U. Epilepsia partialis continua (Kozevnikov-Epilepsie). Fortschr Neurol Psychiatr 1974; 42: 65-212.

6 Juul-Jensen P, Denny-Brown D. Epilepsia partialis continua. Arch Neurol 1966;15;563-86

7 Meienberg $\mathrm{O}$ and Karbowski K. Die Epilepsia partialis continua Kozevnikov. Dtsch Med Wochenschr 1977;102 781-4.

8 Andermann F, Lugaresi E, Dvorkin G, Montagna P. Malignant migraine: the syndrome of prolonged classical migraine migiesia migraine, epilepsia partialis continua, and repeated strokes; a clinically characteristic disorder probable due to mitochondrial encephalopathy. Funct Neurol 1986;1

9 Engel J, Ludwid B, Fetell M. Prolonged partial complex status epilepticus: EEG and behavioral observations. status epilepticus: EEG
Neurology 1978;28:863-9.

10 Knopman D, Margolis G, Reeves A. Prolonged focal epilepsy and hypoxemia as a case of focal brain damage: a case study. Ann Neurol 1977;1:195-8.

11 Duncan R, Patterson J, Hadley D, Bone I. Unilateral cerebellar damage in focal epilepsy. $\mathcal{F}$ Neurol Neurosurg Psychiatry 1990;53:436-7.

12 Scholtes F, Renier W, Meinardi H. Generalized convulsive status epilepticus: causes, therapy and outcome in 346 patients. Epilepsia 1994;35:1104-12.

13 Scholtes F, Reni W, Meinardi H. Generalized convulsive status epilepticus. Pathophysiology and treatment. Pharmacy World and Science 1993;15:17-28.

14 Bancaud J, Bonis A, Trottier S, Talairach J, Dulac O L'epilepsie partielle continue: syndrome et maladie. Rev Leurol (Paris) 1982;138:803-14. 